\title{
Quantitative Analysis of Acid-Base Disorders in Patients with Acute on Chronic Respiratory Failure and Acute Respiratory Distress Syndrome. A Prospective, One Center Study
}

\author{
Claude Guérin ${ }^{1,2,3, *}$, Frédérique Bayle ${ }^{1,2}$, Bernard Poggi ${ }^{2,4}$, Michele Germain-Pastene ${ }^{2,5}$, \\ Pascale Nesme ${ }^{6}$, Gaël Bourdin ${ }^{6}$ and Jean-Christophe Richard ${ }^{1,2,3}$
}

\author{
${ }^{1}$ Service de Réanimation Médicale, Hôpital de la Croix Rousse, Lyon, France; ${ }^{2}$ Université de Lyon, Lyon, France; \\ ${ }^{3}$ Creatis, INSERM U630, Lyon, France; ${ }^{4}$ Laboratoire de Biochimie, Hôpital de la Croix Rousse, Lyon, France; \\ ${ }^{5}$ Laboratoire d'Explorations Fonctionnelles Respiratoire, Hôpital de la Croix Rousse, Lyon, France and ${ }^{6}$ Service de \\ Pneumologie, Hôpital de la Croix Rousse, Lyon, France
}

\begin{abstract}
Background: With Stewart approach, pH depends on strong ion difference (SIDe), $\mathrm{PaCO}_{2}$ and non volatile weak acids (Atot). This approach might detect complex acid-base disorders undetected by conventional approach. We designed this study to describe acid-base disorders with both Stew art and conventional approaches and to compare the diagnostic performance in patients with chronic respiratory failure (CRF) in acute respiratory failure (ARF) or with acute respiratory distress syndrome (ARDS) because they are expected to have complex acid-base disorders. Conventional approach was based on standardized base excess (SBE), bicarbonate $\left(\mathrm{HCO}_{3}^{-}\right)$and anion gap (AG). Working hypotheses were that in CRF, metabolic alkalosis was associated with respiratory acidosis and in ARDS, Stewart approach was able to identify metabolic acidosis not detected with the conventional approach.

Methods: Observational study in a medical ICU of a University hospital on 36 patients with obstructive CRF (CRFo), 36 with non obstructive CRF (CRFno) and 28 with ARDS prospectively included over 8 months. Measurements were performed on ICU admission, day 1 and day 2 after admission.

Results: Metabolic alkalosis occurred in less than 5\% of samples in CRF patients and in 0 ARDS patient. As compared to CRF patients, ARDS patients exhibited lower SBE, higher AG, lower SIDe and lower Atot. In ARDS, low SIDe was primarily due to elevated unidentified anions. Hypoalbuminemia was present in more than $75 \%$ of patients without difference between groups. Normal values of $\mathrm{SBE}, \mathrm{HCO}_{3}{ }^{-}$and $\mathrm{AG}$ were very common. Stewart approach detected low SIDe in $13 \%$ of samples with normal SBE, in $13.8 \%$ of samples with normal $\mathrm{HCO}_{3}{ }^{-}$, and in $11 \%$ of samples with normal AG corrected for abnormal albumin concentration, without difference between CRF and ARDS.

Conclusions: In these selected critically ill patients, Stewart approach exceeded the diagnostic performance of the conventional approach even when AG corrected was taken into account. Further studies in CRF patients with chronic hypercapnia and elevated bicarbonatemia are required to assess the incidence of associated metabolic alkalosis.
\end{abstract}

Key Words: Anion gap, base excess, bicarbonate, acid-base disorder, chronic obstructive pulmonary disease, acute respiratory distress syndrome, acute respiratory failure.

\section{INTRODUCTION}

In the classic understanding of acid-base disorders based on the Henderson Hasselbach equation, bicarbonate $\left(\mathrm{HCO}_{3}{ }^{-}\right)$ buffer is central and plasma $\mathrm{pH}$ dependent on both $\mathrm{pK}$ of $\mathrm{HCO}_{3}{ }^{-}$and plasma $\left[\mathrm{HCO}_{3}{ }^{-}\right] /\left[\mathrm{PaCO}_{2} \times 0.03 \mathrm{ml} / \mathrm{mmHg}\right]$ ratio. This approach may be limited in complex situations, such as respiratory acidosis combined to metabolic acidosis or to metabolic alkalosis in which the distinction between primary and secondary disorders may be difficult. Furthermore, this method does not describe the disorders in quantitative terms. The determination of Base Excess (BE) [1] and Anion Gap

*Address correspondence to this author at the Service de Réanimation Médicale, Hôpital de la Croix Rousse, 103 Grande Rue de la Croix Rousse, 69004 Lyon, France; Tel: 33 (0) 472071 762; Fax: 33 (0) 472071 774; E-mail claude.guerin@chu-lyon.fr
(AG) [2] has provided the conventional approach with some quantification of the disorder. However, AG is underestimated in case of severe hypoalbuminemia [3], which is very common in ICU patients. An alternative concept has been developed by Stewart [4] to overcome the above limitations of the classic approach. According to this theory, plasma $\mathrm{pH}$ depends on 3 independent variables, namely $\mathrm{PaCO}_{2}$, total nonvolatil weak acids plasma concentration (Atot) and strong ion difference (SID), which is the difference between the sum of the strong cations and the sum of the strong anions. With this approach, acid-base disorders fit into 6 categories [5]. Some clinical studies, done in unselected ICU patients, have found that the clinical performance of the Stewart approach was greater than $\mathrm{HCO}_{3}^{-}$and $\mathrm{BE}[5,6]$ but the difference did no longer occur when AG corrected for albuminemia was taken into account [6]. 
To our knowledge, acid-base disorders occurring in acute $(\mathrm{ARF})$ on chronic respiratory failure $(\mathrm{CRF})$ or acute respiratory distress syndrome (ARDS) have not been specifically studied with the Stewart approach. These situations are expected to be not as simple as a pure acute respiratory acidosis or an acute hypoxemia. In ARF on CRF the metabolic compensation, which results in elevated plasma $\mathrm{HCO}_{3}{ }^{-}$, is overwhelmed and acute respiratory acidosis occurs. A metabolic alkalosis may be associated with respiratory acidosis [7] promoted by concomitant diuretic therapy for example. The detection of this metabolic alkalosis has to be taken into account in the ventilatory settings selection to limit the risk of a severe mixed respiratory and metabolic alkalemia, which may promote seizures and/or arrhythmias. Complex acidbase disorders are expected in the ARDS, for at least three reasons. First, severe sepsis or septic shock, which is a leading cause of ARDS, is associated with metabolic acidosis [810]. Second, current recommendations to use mechanical ventilation with low tidal volume [11] would favour concomittant respiratory acidosis. Third, hypoalbuminemia, which is very frequent in ARDS [12], may hidden metabolic acidosis that may be detected with the traditional approach. Therefore, we conducted this prospective study in patients admitted to our ICU for ARF on CRF or ARDS with the following two aims: 1) to describe the acid-base disorders with both Stewart and conventional approaches in these patients on admission and during the early days of their ICU stay, 2) to compare the diagnostic performance of both conventional and Stewart approaches. Our working hypotheses were twofold: 1) in patients with ARF on CRF, metabolic alkalosis was associated with respiratory acidosis, and 2) in patients with ARDS, the Stewart approach was able to identify metabolic acidosis not detected with the conventional approach.

\section{PATIENTS}

This was a prospective study. From $1^{\text {st }}$ November 2006 to $30^{\text {th }}$ June 2007, all patients admitted to our 14-bed respiratory ICU were screened for inclusion. Patients of both genders were included if they met at ICU admission all the following criteria: 1) ARF defined as an increase in breathing discomfort relative to baseline condition and $\mathrm{PaCO}_{2}>45$ $\mathrm{mm} \mathrm{Hg}$ and/or $\mathrm{pH}<7.36$ and/or $\mathrm{PaO}_{2}<55 \mathrm{~mm} \mathrm{Hg}, 2$ ) age $\geq$ 18 years, 3) arterial blood sample tested for complete biochemical analysis on admission and at least on the subsequent two days. Patients were excluded if biochemical analysis was not performed on admission. In addition, eight healthy non smoker volunteers were used to establish the normal range of the acid-base variables in the laboratory. The protocol (EudraCT number 2007-A00495-48) was approved by the local ethics committee (CPP Sud Est III number 2007-030-B). Since procedures were part of the usual diagnostic management, informed consent from patients was waived.

\section{METHODS}

\section{Measurements}

Routine management of ARF on admission in our ICU includes arterial blood withdrawal for measurement of arterial $\mathrm{pH}, \mathrm{PaO}_{2}, \mathrm{PaCO}_{2}$, plasma electrolytes, albumin, total protein and lactate. Samples are systematically repeated daily at 6 a.m. for same biochemistry and additional blood samples may be drawn if indicated. For present study, were retained those results pertaining to the samples performed at ICU admission and at 6 a.m. of the first and second days of the ICU stay. In the normal volunteers, arterial blood was sampled in the post-prandial period. After withdrawn, blood was sent immediately to the clinical biochemistry laboratory. Arterial $\mathrm{pH}$ ( $\mathrm{pH}$ electrode), $\mathrm{PaO}_{2}$ (Clarke's electrode), $\mathrm{PaCO}_{2}\left(\mathrm{PCO}_{2}\right.$ electrode) were measured using Omni $\mathrm{S}$ device (Roche Diagnostics France, Meylan, France). Sodium $\left(\mathrm{Na}^{+}\right)$, potassium $\left(\mathrm{K}^{+}\right)$and chloride $\left(\mathrm{Cl}^{-}\right)$were determined with ion selective using an indirect potentiometric method, calcium $\left(\mathrm{Ca}^{++}\right)$with the Ortho Cresol Phtalein photometric method, phosphate $\left(\mathrm{PO}_{4}^{--}\right)$with the phosphomolybdate method, magnesium $\left(\mathrm{Mg}^{++}\right)$with methylthymol blue complexometric procedure, albumin with the bromocresol purple dye-binding method, total protein with the Biuret method, lactate with a method which employs the oxidation of lactate to pyruvate, all of these using the Dimension RXL analyzer (Dade Behring Inc., Newark, DE, USA).

\section{Calculated Variables}

From the measured $\mathrm{pH}$ and $\mathrm{PaCO}_{2}$, the values of $\mathrm{HCO}_{3}$ and $\mathrm{BE}$ (plasma) were calculated using the Henderson- Hasselbalch and Van Slyke equations, respectively [13]. Standardized BE (SBE) was computed according to Wooten [14, 15]:

$\mathrm{SBE}=\left(\mathrm{HCO}_{3}{ }^{-}-24.4\right)+[(8.3 \times$ albumin $\times 0.15)+(0.29 \times$ $\left.\left.\mathrm{PO}_{4}^{--} \times 0.32\right)\right] \times(\mathrm{pH}-7.4)$

with albumin in $\mathrm{g} / \mathrm{dL}$ and $\mathrm{PO}_{4}{ }^{--}$in $\mathrm{mg} / \mathrm{dL}$.

Albumin ${ }^{-}$and inorganic phosphate $\left(\mathrm{Pi}^{-}\right)$were calculated from the measured values of albumin, $\mathrm{PO}_{4}{ }^{--}$and $\mathrm{pH}$ [5]:

$\operatorname{albumin}^{-}(\mathrm{mM})=$ albumin $\mathrm{x}(0.123 \mathrm{x} \mathrm{pH}-0.631)$

$\mathrm{Pi}^{-}(\mathrm{mM})=\mathrm{PO}_{4}^{--} \times(0.309 \times \mathrm{pH}-0.469)$

AG was calculated as follows:

$\mathrm{AG}(\mathrm{mM})=\left(\mathrm{Na}^{+}+\mathrm{K}^{+}\right)-\left(\mathrm{Cl}^{-}+\mathrm{HCO}_{3}{ }^{-}\right)$

AG was corrected for the effect of abnormal albumin concentration [3]:

AG corrected $(\mathrm{mM})=\mathrm{AG}+0.25$ (normal albumin observed albumin) (in $\mathrm{g} / \mathrm{L}$ )

Apparent SID (SIDa) was computed as [4]:

$\operatorname{SIDa}(\mathrm{mM})=\left(\left[\mathrm{Na}^{+}\right]+\left[\mathrm{K}^{+}\right]+\left[2 \mathrm{x} \mathrm{Ca}^{++}\right]+\left[2 \mathrm{x} \mathrm{Mg}^{++}\right]\right)-$ $\left(\left[\mathrm{Cl}^{-}+\right.\right.$lactate $\left.]\right)$

SIDe was calculated as follows [5]:

SIDe $(\mathrm{mM})=\mathrm{HCO}_{3}^{-}+$albumin $^{-}+\mathrm{Pi}$

The strong ion gap (SIG) was obtained as [16]:

$\mathrm{SIG}=\mathrm{SIDa}-\mathrm{SIDe}$

A positive value of SIG must reflect unmeasured anions (sulphate, keto acids, citrate, pyruvate, acetate, gluconate) whereas a negative value of SIG must reflect unmeasured cations.

Atot was computed as:

Atot $=$ albumin $^{-}+\mathrm{Pi}^{-}$ 
Unidentified strong anions (XA) were computed as follows [5]:

$\mathrm{XA}(\mathrm{mM})=\left(\left[\mathrm{Na}^{+}\right]+\left[\mathrm{K}^{+}\right]+\left[2 \mathrm{x} \mathrm{Ca}^{++}\right]+\left[2 \mathrm{x} \mathrm{Mg}^{++}\right]\right)-\left[\mathrm{Cl}^{-}\right]$ - SIDe

$\mathrm{XA}$ and $\mathrm{Cl}$ were corrected for water excess/deficit by multiplying their observed values by a correcting factor $\left(\mathrm{Na}^{+}\right.$ normal $/ \mathrm{Na}^{+}$observed) [5].

At time of ICU admission the following variables were recorded: age, gender, simplified acute physiology score II [17], origin, context of admission, sepsis or septic shock [18], number of organ system dysfunction [19]. Were also recorded at time of ICU admission, at day 2 and day 3 the amount and type of fluid (crystalloid, colloid, albumin, red blood cells, and other fluids including fresh frozen plasma and platelets) used for resuscitation within the hours before the blood was sampled, and the presence of mechanical ventilation either invasive or non invasive.

\section{Data Analysis}

CRF was defined from clinical history and arterial blood gas data before ICU stay and/or pulmonary function tests obtained either before or after ICU stay. CRF was separated into obstructive (CRFo) and non obstructive (CRFno) groups. The former comprised of chronic obstructive pulmonary disease (COPD) and other obstructive disorders, and the latter of neuromuscular and non neuromuscular (kyphoscoliosis, tuberculosis sequelae, interstitial fibrosis, hypoventilation-obesity syndrome) respiratory disorders. COPD was defined by GOLD criteria [20]. ARDS was defined by the consensus conference criteria [21] (acute onset of ARF requiring tracheal intubation, bilateral lung infiltrates on frontal chest-X-ray, $\mathrm{PaO}_{2} / \mathrm{FIO}_{2} \leq 200 \mathrm{~mm} \mathrm{Hg}$, no argument for an increased left atrial pressure).

Acid-base diagnosis was performed in each patient using conventional analysis [22] and physico-chemical analysis [5]. For the latter, the diagnostic categories defined by Fencl et al. [5] were used.

\section{Statistical Analysis}

Values are expressed as median $\left(25^{\text {th }}-75^{\text {th }}\right.$ percentiles $)$. Normal ranges were established from the values obtained in the 8 healthy volunteers. The normal distribution of the variables in the healthy volunteers was verified by the AndersonDarling test. Abnormal values for the definition of every acid-base disorder were taken as those lower or greater by 3 SD than the mean value of the healthy volunteers. Age, SAPS II and number of organ dysfunction were compared between CRFo, CRFno and ARDS groups with the non parametric Kruskal-Wallis test and the Dunn-Sidak test for post-hoc pairwise comparisons. To verify if infusion and ventilation are confounding factors one-way ANOVA for repeated measures was performed on patients that were infused or ventilated. Pearson's chi-Square test was used to compare counts and proportions. If the chi-square test was not acceptable (expected count in each cell must be $>1$ and/or expected count $>5$ in more than $20 \%$ of the cells), the Fisher's exact probability was computed. The correlations between variables were assessed by least-squares regression analysis [23]. The statistical level of significance was set at P $<0.05$. The statistical analysis was made by SPSS for win- dows software (version 15.0.1) (2006 SPSS Inc., Chicago, IL, USA) except for the Anderson-Darling test, which was assessed by using the 2007 Minitab software (version 15.1.1.0) (Minitab, Inc.) and for the Fisher's exact test which was computed on line (http://www.u707.jussieu.fr/biostatgv/ fisher_fonction.php).

\section{RESULTS}

\section{Cohort}

Two-hundred ninety-three patients were admitted to our ICU during the study period. Among them, 101 were eligible to the present study: 36 with CRFo, 37 with CRFno and 28 with ARDS (Table 1). One CRFno patient was excluded for lack of data and, hence, 100 patients were retained in the analysis. Two patients, one with CRFo and one with CRFno, who miss day 3 blood sample, were kept in the analysis, which was, therefore, performed on a total of 298 blood samples. The context of admission was a medical illness in $98 \%$ of the patients without any significant difference between groups. The aetiology of CRFo was COPD in 33 patients and bronchiectasis in 3. CFRno resulted from a non neuromuscular respiratory disease in 18 cases, a neuromuscular respiratory disease in 9 and an obesity-hypoventilation syndrome in 9. The distribution of the precipitating factor of ARF was not significantly different between CRFo and CRFno: pneumonia 12 vs. 8 , non respiratory sepsis 2 vs. 4 , bronchitis 3 vs. 0 , pulmonary embolism 2 vs. 2, left ventricle failure 4 vs. 3 , septic shock 1 vs. 0 , other or unknown 12 vs. 19 (Fisher's exact test, $\mathrm{P}=0.3023$ ). In all but 3 patients, the ARDS resulted from a direct lung injury. The ARDS patients had a greater number of organ dysfunction and were in severe sepsis or septic shock and received vasoactive support and renal replacement therapy more frequently than patients in each of CRF groups (Table 1).

\section{Acid-Base Abnormalities}

The median (IQR) and mean (SD) values of acid-base variables are shown in Table $\mathbf{2}$ for healthy volunteers. The median (IQR) values of acid-base variables are displayed in Table 3 for patients on ICU admission. The calculated acidbase variables did not significantly differ between CRFo and CRFno (Table 3). By contrast, all of these, except for AG and corrected chloride, were significantly different between ARDS and CRF groups (Table 3). SIDe was significantly lower in the ARDS group than in each of CRF groups as a result from higher XA. Atot was lower in the ARDS group as a result from lower plasma albumin level.

The two acid-base variables which significantly changed over time were $\mathrm{PaCO}_{2}$ and phosphate. $\mathrm{PaCO}_{2}$ and phosphate in CRFo and phosphate in CRFno patients significantly declined between. No change occurred over time in ARDS patients.

Only three pure acid-base derangements were correctly identified over the 298 samples by the conventional approach on admission: metabolic acidosis in 1 ARDS patient and respiratory acidosis in 1 CRFno patient and in 1 ARDS patient.

The diagnostic categories of acid-base derangements according to the Stewart approach in the 100 patients on admission are shown in Table 4. Forty-five patients (45\%) had 
Table 1. Patients Data Entry

\begin{tabular}{|c|c|c|c|c|}
\hline Variables & CRFo $(n=36)$ & CRFno $(n=36)$ & $\operatorname{ARDS}(\mathrm{n}=28)$ & P Value \\
\hline Male Gender, n (\%) & $27(75)$ & $19(53)$ & $18(64)$ & $0.145 * *$ \\
\hline $\begin{array}{c}\text { Origin, } \mathrm{n}(\%) \\
\text { Other hospital } \\
\text { Home } \\
\text { Ward } \\
\text { Emergency }\end{array}$ & $\begin{array}{c}11(30.6) \\
0(0) \\
13(36) \\
12(33.3)\end{array}$ & $\begin{array}{l}10(28) \\
1(2.8) \\
13(36) \\
12(33)\end{array}$ & $\begin{array}{c}14(50) \\
0(0) \\
6(21) \\
8(29)\end{array}$ & $0.455 * * *$ \\
\hline Age $*$, years & $72(62-78)$ & $71(55-80)$ & $64(55-74)$ & $0.281 * * * *$ \\
\hline SAPS II *, score & $41(34-53)$ & $41(26-51) \dagger$ & $55(39-71)$ & $0.040 * * * *$ \\
\hline $\mathrm{ODF}^{*}$, number & $1(1-2) \dagger$ & $1(1-2) \dagger$ & $2(2-3)$ & $0.0001 * * * *$ \\
\hline Septic shock or severe sepsis, n (\%) & $10(28)$ & $10(28)$ & $21(75)$ & $0.0001 * *$ \\
\hline Vasoactive agents, n (\%) & $8(22)$ & $9(25)$ & $19(68)$ & $0.0001 * *$ \\
\hline Renal replacement therapy, n (\%) & $1(3)$ & $2(6)$ & $7(25)$ & $0.0114 * * *$ \\
\hline
\end{tabular}

$*$ median $\left(25^{\text {th }}-75^{\text {th }}\right.$ percentile), ** Chi-square test, *** Fisher's exact test, $* * * *$ Kruskal-Wallis test, $\dagger \mathrm{P}<0.05$ vs. ARDS group (Dunn-Sidak test).

SAPS = simplified acute physiology score, ODF = organ dysfunction (including respiratory).

Table 2. Acid base Variables in the 8 Healthy Volunteers

\begin{tabular}{|c|c|c|}
\hline \multicolumn{3}{|c|}{ Measured Variables } \\
\hline Potassium $(\mathrm{mM})$ & $3.9(0.3)$ & $3.8(3.7-4.1)$ \\
\hline Calcium (mM) & $2.3(0.1)$ & $2.3(2.3-2.3)$ \\
\hline Chloride (mM) & $103(2)$ & $103(102-105)$ \\
\hline Phosphate (mM) & $1.0(0.1)$ & $1.0(0.9-1.1)$ \\
\hline Total protein $(\mathrm{g} / \mathrm{L})$ & $73(3)$ & $73(72-74)$ \\
\hline Albumin $(\mathrm{g} / \mathrm{L})$ & $42(4)$ & $44(38-44)$ \\
\hline $\mathrm{pH}$ & $7.42(0.03)$ & $7.41(7.40-7.42)$ \\
\hline \multicolumn{3}{|c|}{ Calculated Variables (mM) } \\
\hline Bicarbonate & $25(2)$ & $25(24-25)$ \\
\hline Standardized base excess & $0.5(2.4)$ & $0.3(-0.6-0.7)$ \\
\hline Albumin ${ }^{-}$ & $12(1)$ & $12(11-13)$ \\
\hline Phosphate $^{-}$ & $1.9(0.2)$ & $1.9(1.8-2.0)$ \\
\hline Anion Gap & $15(2)$ & $15(14-17)$ \\
\hline Anion Gap corr & $15(2)$ & $15(14-17)$ \\
\hline SIDa & $46(2)$ & $47(45-48)$ \\
\hline SIDe & $39(3)$ & $39(37-39)$ \\
\hline
\end{tabular}

$\mathrm{SIDa}=$ apparent strong ion dissociation, $\mathrm{SIDe}=$ effective strong ion dissociation, $\mathrm{XA}=$ undosed anions. 
Table 3. Acid-Base Variables in Patients at Time of ICU Admission

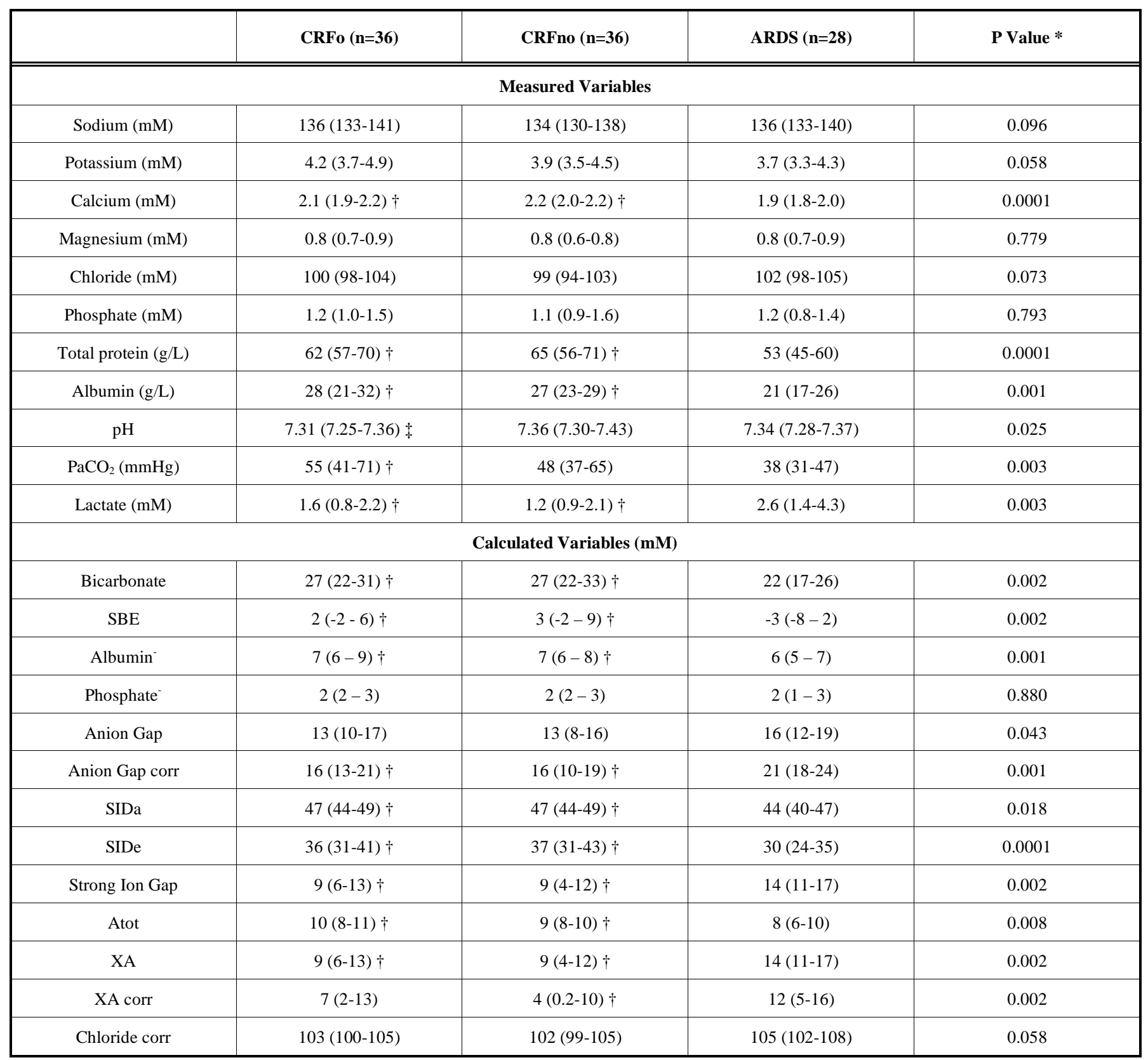

Values are median $\left(25^{\text {th }}-75^{\text {th }}\right.$ percentile $)$.

$*$ Kruskal-Wallis test, $\dagger \mathrm{p}<0.05$ vs. ARDS group, $\ddagger \mathrm{p}<0.05$ vs. CRFno group (Dunn-Sidak test)

$\mathrm{SBE}=$ standardized base excess, $\mathrm{SIDa}, \mathrm{SIDe}=$ apparent and effective strong ion dissociation, $\mathrm{XA}=$ undosed anions.

elevated $\mathrm{PaCO}_{2}$ with a significantly greater proportion in CRFo and CRFno than in ARDS patients, a pattern that did not change over time (Fig. 1). Three patients (3\%) had low $\mathrm{PaCO}_{2}$ value (Table 4). Twelve patients $(12 \%)$ had dilution acidosis, without difference between groups (Table 4). Ten patients $(10 \%)$ had a low SIDe due to elevated XA with a greater proportion in ARDS patients (Table 4). The same was true over time (Fig. 1). Three patients, all with CRF, had high SIDe, which was essentially due to low chloride. Thirty-five $(35 \%)$ patients had elevated phosphatemia without any difference between groups (Table 4). Over time, however, the proportion of hyperphosphatemia significantly declined among CRFo and CRFno patients (Fig. 1). Hypoal- buminemia was present in 88 patients $(88 \%)$ on admission with a greater proportion in ARDS than in CRFo patients (Table 4), a difference between groups that persisted over time (Fig. 1). Finally, 15 patients $(15 \%)$ had a low phosphatemia on admission with no difference between groups (Table 4) and over time.

ARDS patients were more frequently infused with crystalloid and colloid at day 1, with albumin and blood at day 2 and day 3 than patients in each of the CRF groups (Table 5). Among the infused patients, the amount of crystalloid, but not that of colloids or other fluids, was significantly greater at day 3 than at day 1 and day 2 in each group (not shown). At day $1,100 \%$ of the ARDS patients received mechanical 
Table 4. Diagnostic Categories of Acid Base Disorders According to the Stewart Approach in Patients on Admission

\begin{tabular}{|c|c|c|c|c|}
\hline & $\begin{array}{c}\text { CRFo } \\
(n=36)\end{array}$ & $\begin{array}{c}\text { CRFno } \\
(n=36)\end{array}$ & $\begin{array}{l}\text { ARDS } \\
(n=28)\end{array}$ & P Value \\
\hline \multicolumn{5}{|c|}{ Respiratory Disorders } \\
\hline $\mathrm{PaCO}_{2}$ high & $22(61.1)$ & $17(47.2)$ & $6(21.4)$ & $0.006 *$ \\
\hline $\mathrm{PaCO}_{2}$ low & $1(2.8)$ & $1(2.8)$ & $1(3.6)$ & $1.00 \dagger$ \\
\hline \multicolumn{5}{|c|}{ Non Respiratory disorders } \\
\hline \multicolumn{5}{|l|}{ SIDe low } \\
\hline Sodium low & $4(11.1)$ & $3(8.3)$ & $5(17.9)$ & $0.4685 \dagger$ \\
\hline Chloride high & 0 & 0 & 0 & \\
\hline Undosed anions & $3(8.3)$ & $1(2.8)$ & $6(21.4)$ & $0.02959 \dagger$ \\
\hline \multicolumn{5}{|l|}{ SIDe high } \\
\hline Sodium high & 0 & 0 & 0 & \\
\hline Chloride low & $1(2.8)$ & $2(5.6)$ & 0 & $0.7762 \dagger$ \\
\hline \multicolumn{5}{|c|}{ Non volatile weak acids } \\
\hline Albumin high & 0 & 0 & 0 & \\
\hline Phosphate high & $13(36.1)$ & $13(36.1)$ & $9(32.1)$ & $0.9326 *$ \\
\hline Albumin low & $23(63.9)$ & $30(83.3)$ & $25(89.3)$ & $0.032 *$ \\
\hline Phosphate low & $6(16.7)$ & $5(13.9)$ & $4(14.3)$ & $1.00 \dagger$ \\
\hline
\end{tabular}

$*$ Pearson's chi square, $\dagger$ Fisher's exact test.

Number of patients (\% in group).

$\mathrm{CRF}=$ chronic respiratory failure, $\mathrm{o}=$ obstructive, no $=$ non obstructive, $\mathrm{ARDS}=$ acute respiratory distress syndrome, $\mathrm{SIDe}=$ effective strong ion difference .

Table 5. Rates of Fluid Resuscitation Among Groups Over Time

\begin{tabular}{|c|c|c|c|c|}
\hline & $\begin{array}{l}\text { CRFo } \\
(n=36)\end{array}$ & $\begin{array}{c}\text { CRFno } \\
(n=36)\end{array}$ & $\begin{array}{l}\text { ARDS } \\
(n=28)\end{array}$ & P Value \\
\hline \multicolumn{5}{|l|}{ Day 1} \\
\hline Cristalloids & $5(13.9)$ & $3(8.3)$ & $10(35.7)$ & $0.018^{*}$ \\
\hline Colloids & $4(11.1)$ & $3(8.3)$ & $9(32.1)$ & $0.031 *$ \\
\hline Albumin & 0 & 0 & $2(7.1)$ & $0.076^{*}$ \\
\hline Other & 0 & 0 & $1(3.6)$ & $1.000^{*}$ \\
\hline \multicolumn{5}{|l|}{ Day 2} \\
\hline Cristalloids & $17(47.2)$ & $16(44.4)$ & $16(57.1)$ & $0.58 \dagger$ \\
\hline Colloids & $10(27.8)$ & $9(24.9)$ & $17(60.7)$ & $0.006 \dagger$ \\
\hline \multicolumn{5}{|l|}{ Day 3} \\
\hline Cristalloids & $14(38.9)$ & $16(44.4)$ & $17(60.7)$ & $0.15 \dagger$ \\
\hline Colloids & $4(11.1)$ & $4(11.1)$ & $8(28.5)$ & $0.126^{*}$ \\
\hline Albumin & $5(13.9)$ & $1(2.8)$ & $13(46.4)$ & $0.00003 *$ \\
\hline Blood & $2(5.5)$ & 0 & $6(21.4)$ & $0.0058^{*}$ \\
\hline Other & $1(2.8)$ & 0 & $3(10.7)$ & $0.095^{*}$ \\
\hline
\end{tabular}

Number of patients (\% in group).

$*$ Fisher's exact test, $\uparrow$ Pearson chi-square.

$\mathrm{CRF}=$ chronic respiratory failure, $\mathrm{o}=$ obstructive, no $=$ non obstructive, $\mathrm{ARDS}=$ acute respiratory distress syndrome 

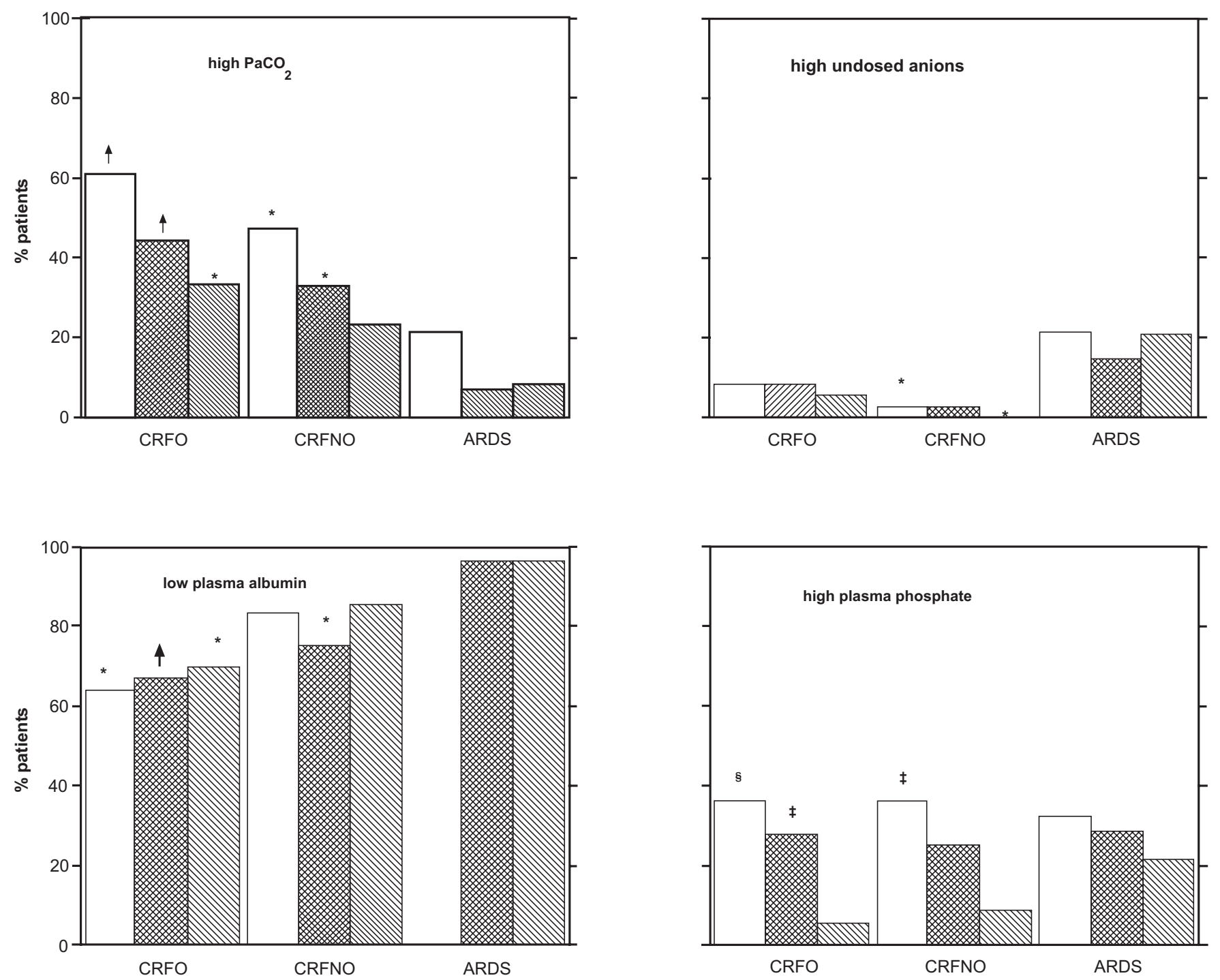

Fig. (1). Proportions of patients with high $\mathrm{PaCO}_{2}$, elevated undosed anions, low plasma albumin and high plasma phosphate in patients with obstructive chronic respiratory failure ( $\mathrm{CRFo}$ ), non obstructive chronic respiratory failure (CRFno) and acute respiratory distress syndrome (ARDS) at day 1 (white bars), day 2 (crossed bars) and day 3 (hatched bars). $* \mathrm{P}<0.05$ and $\dagger \mathrm{P}<0.01$ vs. ARDS, $\ddagger \mathrm{P}<0.05$ and $\S \mathrm{p}<0.001$ vs. day 3 . High or low values were defined as 3 SD above or below the mean values found in the 8 healthy volunteers.

ventilation vs. $50 \%$ in $\mathrm{CRFo}(\mathrm{P}<0.00001)$ and $71.4 \%$ in CRFno $(\mathrm{P}<0.01)$. At day 2 , these proportions were $100 \%$ vs. $61 \%(\mathrm{P}<0.001)$ and $81 \%(\mathrm{P}<0.05)$. At day 3 , the corresponding rates were $100 \%$ vs. $66.6 \%(\mathrm{P}<0.01)$ and $86.1 \%$ $(\mathrm{P}>0.05)$. The rates were not significantly different between CRFo and CRFno. Time and infusion or mechanical ventilation had no significant effect on SBE, SIDe, SIDa, SIG, Atot, AG, AGcorr, XA and XAcorr between patients groups (one-way repeated measures ANOVA). Time but not infusion or mechanical ventilation had a significant effect on $\mathrm{PaCO}_{2}$ between patients groups.

The pattern of acid-base diagnostic categories according to Stewart approach in the $69(69 \%)$ with normal SBE, in the 65 patients $(65 \%)$ with normal bicarbonatemia and for the 73 patients $(73 \%)$ with normal AG corrected on admission was not different between groups (Table 6).

The correlations between SIDe and SBE, between $\mathrm{HCO}_{3}{ }^{-}$ and SBE, between SIG and AG corrected, and between SIDe and $\mathrm{HCO}_{3}{ }^{-}$were significant in both healthy volunteers (Fig. 2) and patients (Fig. 3).

\section{DISCUSSION}

The main findings of present study were that: 1) in CRF patients with ARF, high values of SIDe were very uncommon, in both obstructive and non obstructive groups, and so was the occurrence of concomittant metabolic alkalosis, 2) ARDS patients quite frequently exhibited low values of SIDe, 3) hypoalbuminemia was very common in the three groups of patients, 4) the Stewart approach improved slightly the diagnostic performance of the conventional approach.

It was rather surprising to find out in present study that the conventional approach, such as that proposed by Kellum [22], identified simple acid-base disorders in $1 \%$ of the samples. By using normal ranges of present study in healthy volunteers did not improve the diagnostic performance of the conventional procedure. This finding underscores the fact 
Table 6. Diagnostic Categories of Acid-Base Disorders According to the Stewart Approach in Patients with Normal SBE, Normal $\mathrm{HCO}_{3}$ and Normal AG on Admission

\begin{tabular}{|c|c|c|c|}
\hline & $\begin{array}{l}\text { Normal SBE } \\
\quad(n=69)\end{array}$ & $\begin{array}{l}\text { Normal } \mathrm{HCO}_{3} \\
\quad(n=65)\end{array}$ & $\begin{array}{l}\text { Normal AG Corrected } \\
\qquad(\mathbf{n}=73)\end{array}$ \\
\hline $\mathrm{PaCO}_{2}$ high & 44.9 & 44.6 & 52.1 \\
\hline $\mathrm{PaCO}_{2}$ low & 1.4 & 0 & 1.4 \\
\hline \multicolumn{4}{|l|}{ Non Respiratory Disorders } \\
\hline \multicolumn{4}{|l|}{ SIDe low } \\
\hline Sodium low & 13.0 & 12.3 & 9.6 \\
\hline Chloride high & 2.8 & 0 & 0 \\
\hline Undosed anions & 1.4 & 1.5 & 1.4 \\
\hline Chloride low & 0 & 0 & 0 \\
\hline \multicolumn{4}{|l|}{ Non Volatile Weak Acids } \\
\hline Albumin high & 0 & 0 & 0 \\
\hline Phosphate high & 34.8 & 23.4 & 31.5 \\
\hline Albumin low & 78.3 & 82 & 78.9 \\
\hline Phosphate low & 14.5 & 16.1 & 19.2 \\
\hline
\end{tabular}

Numbers in table are $\%$ of patients in group.

$\mathrm{SBE}=$ standardized base excess, $\mathrm{HCO} 3=$ bicarbonate, $\mathrm{AG}=$ anion gap, SIDe $=$ effective strong ion difference

that in this patient population the acid-base disorders are particularly complex.

Contrary to our initial hypothesis concomittant metabolic alkalosis was discarded in most instances in patients with CRF in present study since it was observed in less than 5\% of the CRF patients: $4.2 \%$ (3/72) at day $1,4.2 \%(3 / 72)$ at day 2 and $2.9 \%(2 / 70)$ at day 3. Holland et al. [7] found that metabolic alkalosis contributed to acute hypercapnic respiratory failure in $71 \%$ of their 14 patients with cystic fibrosis and in $22 \%$ of their 49 COPD patients in acute exacerbation. However, the analysis used in this study was not extensive and, specifically, no correction for hypoalbuminemia, which was significantly different between the two groups, was made. In present investigation, no patient suffered from cystic fibrosis. Furthermore, it should be stressed that in present cohort the mean level of plasma bicarbonate was not markedly elevated in CRFo and CRFno patients and, indeed, was not significantly different from the 8 healthy volunteers. In this connection, all the occurrences of elevated SIDe were observed in those CRF patients who were hypercapnic. In dogs exposed to $\mathrm{CO}_{2}$ for one week, the decrease in plasma $\mathrm{pH}$ was not a prerequisite to the increase in $\mathrm{HCO}_{3}{ }^{-}$reabsorption by the kidney required for sustaining the secondary hyperbicarbonatemia characteristic of chronic hypercapnia [24]. Finally, elevated SIDe was due to hypochloremic alkalosis and not to contraction alkalosis in present study. Further studies should be performed to rule out the issue of metabolic alkalosis associated with respiratory acidosis in a substantial number of patients with chronic hypercapnia and elevated plasma bicarbonate.

In ARDS patients, as in CRF patients, there was a mix of respiratory and non respiratory disorders according to the classification proposed by Fencl et al. [5]. On admission, 22 patients $(22 \%)$ had low SIDe (39.3\% of the ARDS patients vs. $15.3 \%$ of the CRF patients, $P<0.05)$. In 10 of them the low SIDe was due to elevated XA. Elevated lactate (greater than $3 \mathrm{SD}$ above the mean of the healthy volunteers) was responsible for this in 7 patients, namely 1 CRFo, 1 CRFno and 5 ARDS patients. Sepsis was the primary mechanism for elevated lactate because all these 7 patients had severe sepsis or septic shock criteria on admission. Over the 298 samples, a rate of 16.4\% (49/298) of low SIDe was observed (28.6\% in ARDS vs. $11.7 \%$ in CRF patients, $\mathrm{P}<0.001$ ). In 25 of them the low SIDe was due to elevated XA. Elevated lactate (greater than $3 \mathrm{SD}$ above the mean of the healthy volunteers) was responsible for this in 18 patients, namely 4 CRFo, 2 CRFno and 12 ARDS patients. Sepsis was the primary mechanism for elevated lactate because all these 18 patients had severe sepsis or septic shock criteria. Therefore, lactate should be measured systematically on admission in CRF patients in ARF and in ARDS patients. The low SIDe due to low plasma sodium might be related to the perfusion of crystalloids with low SIDe because ARDS and CRFo patients who received the largest amounts of crystalloids had the 

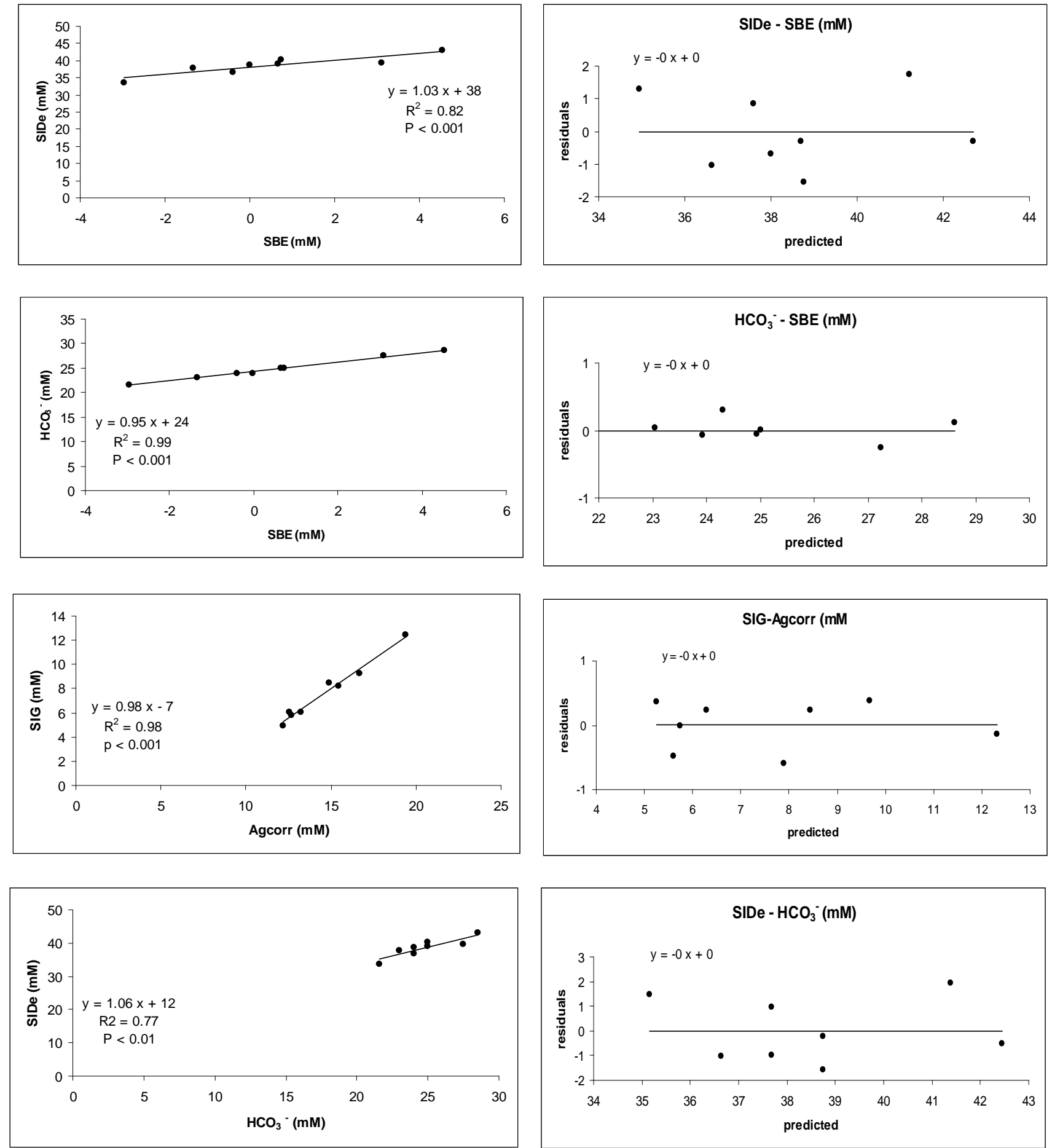

Fig. (2). Relationships between effective strong ion difference (SIDe) and standardized base excess ( $\mathrm{SBE}$ ), $\mathrm{SBE}$ and bicarbonate (HCO ${ }_{3}{ }^{-}$), strong ion gap (SIG) and albumin-corrected anion gap (AGcorr) and SIDe and $\mathrm{HCO}_{3}{ }^{-}$in 8 healthy volunteers. On the left panels the lines are the regression lines over all subjects with their regression equations. Right panels depict the corresponding relationships of the residuals to the expected values.

highest rate of dilution acidosis. However, even though this amount of fluids was received prior to the blood sampling on admission, the time delay between the infusion and blood withdrawal was not controlled.

Present investigation confirms the high prevalence of hypoalbuminemia on ICU admission. This finding has already been described in mixed ICU patients $[5,6]$ and in
ARDS as well [12]. We confirmed that high prevalence of hypoalbuminemia also occurred in patients with CRFno.

In present study, we found a high rate of abnormal values of plasma phosphate, which can contribute to the acid-base imbalances according to Stewart approach. It should be noted that, even though it is frequently observed, the quantitative contribution of this finding to the magnitude of acid- 

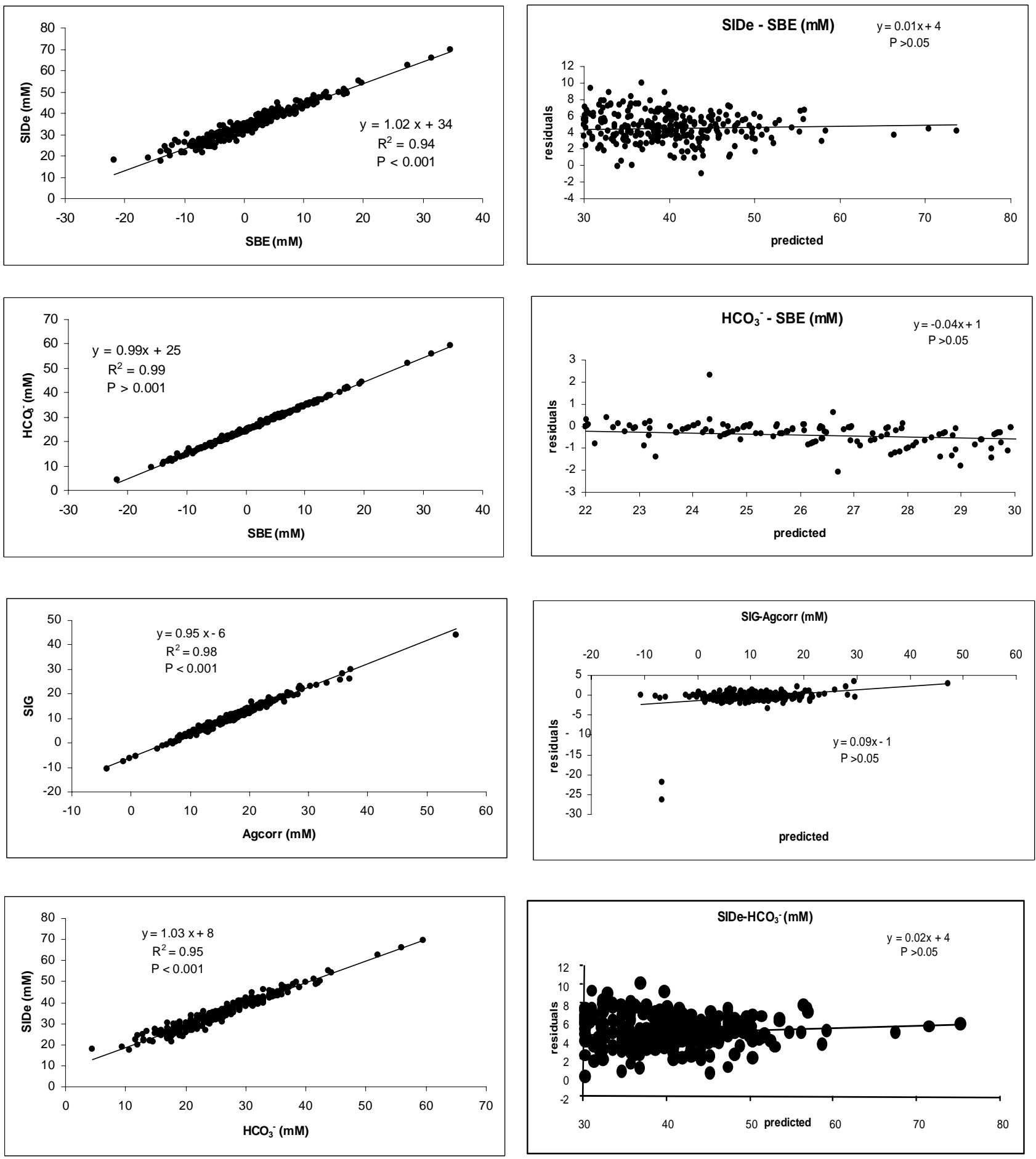

Fig. (3). Relationships between effective strong ion difference (SIDe) and standardized base excess ( $\mathrm{SBE}$ ), $\mathrm{SBE}$ and bicarbonate $\left(\mathrm{HCO}_{3}{ }^{-}\right.$), strong ion gap (SIG) and albumin-corrected anion gap (AGcorr) and SIDe and $\mathrm{HCO}_{3}{ }^{-}$in patients. On the left panels the lines are the regression lines over the all patients with their regression equations. Right panels depict the corresponding relationships of the residuals to the expected values.

base derangement is limited. However, this high rate strongly suggests to measure plasma phosphate on admission and to repeat it over time. One third of present patients had high plasma phosphate on admission, a rate which decreased over time in CRF patients but not in ARDS patients. It has been described that acute reduction of phosphoremia, as it occurs after rapid reduction in $\mathrm{PaCO}_{2}$ and rapid increase in plasma $\mathrm{pH}$ after onset of mechanical ventilation, may lead to severe cardiac arrhythmias and seizures in CRF patients in $\operatorname{ARF}[25,26]$.

In our study, patients were followed up to the first two days of ICU stay. This design has the advantages to collect a greater number of observations and to study the effect of 
time on the acid-base derangements. The drawback of the repeated measures in an observational study like the present one is the lack of control for confounding factors, such as the fluid infused. This latter has, however, been taken into account and discarded by the one-way analysis. In a large number of patients who were prospectively randomized to receive either $4 \%$ human albumin or normal saline for fluid resuscitation in ICU, the main factor which predicted changes in acid-base status over time was the total amount of fluid received rather than its nature [27]. It should be stressed that in this study done over 691 patients, $2.2 \%$ and $3.5 \%$ only had ARDS and COPD, respectively.

Overall, $79 \%$ of patients received mechanical ventilation in present study, a rate which was markedly higher than that of 14\% among the 935 patients of Dubin et al. who reported the largest series to date of ICU patients extensively studied in terms of acid-base status with conventional and Stewart approach [6]. Of notice, this report did not provide the reader with the number of ARDS or CRF patients included. Nevertheless and interestingly, the correlations between SIDe and $\mathrm{BE}, \mathrm{SIG}$ and $\mathrm{AG}$ corrected, and $\mathrm{HCO}_{3}$ and $\mathrm{BE}$ or SIDe were very close in present study done on ARDS and CRF patients, who were frequently mechanically ventilated, and in Dubin's study done on mixed ICU patients who were rarely receiving mechanical ventilation.

Present results of the Stewart approach in normal volunteers are similar to those found in two previous reports $[5,6]$. In present study mean SIDe in normals amounted to $36 \pm 7$ $\mathrm{mM}$, not different from $39 \pm 1$ and $40 \pm 2 \mathrm{mM}$ found by Fencl et al. and Dubin et al. respectively. The corresponding values of SIG were $8 \pm 7,8 \pm 2$ and $2 \pm 2 \mathrm{mM}$, respectively.

In conclusions, we found that: 1) metabolic alkalosis did not contribute to respiratory acidosis in CRF patients significantly, 2) low SIDe was quite frequent in ARDS and CRF patients and primarily due to elevated XA as a result from elevated lactate from severe sepsis/septic shock, 3) Stewart approach was able to detect low SIDe in a substantial number of patients with CRF and ARDS patients which were classified as normal by the conventional approach, 4) in these selected critically ill patients, Stewart approach exceeded the diagnostic performance of the conventional approach even when AG corrected was taken into account.

\section{REFERENCES}

[1] Siggaard-Andersen, O. The acid-base status of the blood, 4th. Munksgaard: Copenhagen, 1974.

[2] Emmett, M.; Narins, R.G. Medicine (Baltimore), 1977, 56, 38-54

[3] Figge, J.; Jabor, A.; Kazda, A.; Fencl, V. Crit. Care Med., 1998, 26, 1807-1810

[4] Stewart, P.A. Can. J. Physiol. Pharmacol., 1983, 61, 1444-1461.

[5] Fencl, V.; Jabor, A.; Kazda, A.; Figge, J. Am. J. Respir. Crit. Care Med., 2000, 162, 2246-2251.

[6] Dubin, A.; Menises, M.M.; Masevicius, M.D.; Moseinco, M.C.; Kutscherauer, D.O.; Ventrice, E.; Laffaire, E.; Estenssoro, E. Crit. Care Med., 2007, 35, 1264-1270.

[7] Holland, A.E.; Wilson, J.W.; Kotsimbos, T.C.; Naughton, M.J. Chest, 2003, 124, 490-493

[8] Kellum, J.A.; Song, M.; Almasri, E. Chest, 2006, 130, 962-967.

[9] Kellum, J.A. Crit. Care Resusc., 2004, 6, 197-203.

[10] Kellum, J.A.; Venkataraman, R. Crit. Care Med., 2002, 30, $1387-$ 1388.

[11] ARDSnet N. Engl. J. Med., 2000, 342, 1301-1308.

[12] Martin, G.S.; Moss, M.; Wheeler, A.P.; Mealer, M.; Morris, J.A.; Bernard, G.R. Crit. Care Med., 2005, 33, 1681-1687.

[13] Siggaard-Andersen, O.; Wimberly, P.D.; Fogh-Andersen, N.; Gothgen, I.H. Scand. J. Clin. Lab. Invest., 1988, 48, 7-15.

[14] Wooten, E.W. Crit. Care, 2004, 8, 448-452.

[15] Wooten, E.W. J. Appl. Physiol., 2003, 95, 2333-2344.

[16] Kellum, J.A.; Kramer, D.J.; Pinsky, M.R. J. Crit. Care, 1995, 10 , 51-55.

[17] Le Gall, J.R.; Lemeshow, S.; Saulnier, F. JAMA, 1993, 270, $2957-$ 2963.

[18] Bone, R.C.; Fisher, C.J., Jr.; Clemmer, T.P.; Slotman, G.J.; Metz, C.A.; Balk, R.A. Crit. Care Med., 1989, 17, 389-393.

[19] Fagon, J.Y.; Chastre, J.; Novara, A.; Medioni, P.; Gibert, C. Intensive Care Med., 1993, 19, 137-144.

[20] National Institute of Health. Global Initiative for Chronic Obstructive Lung Disease. Global Strategy for the Diagnosis, Management and Prevention of Chronic Obstructive Pulmonary Disease. In: NHLBI/WHO workshop report, 1 July 2003 edn (ed. (2003). National Heart, Lung and Blood Institute, Bethesda.

[21] Bernard, G.R.; Artigas, A.; Brigham, K.L.; Carlet, J.; Falke, K.; Hudson, L.; Lamy, M.; Legall, J.R.; Morris, A.; Spragg, R. Am. J. Respir. Crit. Care Med., 1994, 149, 818-824.

[22] Kellum, J.A. Crit. Care, 2005, 9, 500-507.

[23] Hopkins, W.G. Sportscience, 2004, 8, 42-46.

[24] Madias, M.E.; Wolf, C.J.; Cohen, J.J. Kidney Int., 1985, 27, 538543.

[25] Laaban, J.P.; Grateau, G.; Psychoyos, I.; Laromiguiere, M.; Vuong, T.K.; Rochemaure, J. Crit. Care Med., 1989, 17, 1115-1120.

[26] Laaban, J.P.; Marsal, L.; Waked, M.; Vuong, T.K.; Rochemaure, J. Intensive Care Med., 1990, 16, 135-136.

[27] Bellomo, R.; Morimatsu, H.; French, C.; Cole, L.; Stiry, D.; Uchino, S.; Naka, T. Crit. Care Med., 2006, 34, 1-7. 